\title{
Influence of perceived benefits and traceability system on the readiness for Halal Assurance System implementation among food manufacturers
}

\begin{abstract}
Despite consistent monitoring by the Department of Islamic Development Malaysia (JAKIM), the fabrication of fake halal logos and certificates by irresponsible parties continues to challenge the integrity and authenticity of halal products. Malaysia's Halal Certification Procedures (Second Revision, 2012) state that companies operating in Malaysia need to develop their Halal Assurance System (HAS) to strengthen the management of halalrelated matters within organizations. The purpose of this study is to investigate the influence of perceived benefits and traceability practices on readiness for Halal Assurance System (HAS) implementation. The study was conducted through a cross-sectional survey, and simple random sampling was employed. At the end of the data collection period, a total of 139 responses from halal-certified small and medium enterprises (SMEs) were obtained. Structural equation modeling with Amos graphics was used to perform a confirmatory factor analysis (CFA) and a causal path analysis. Results indicate that perceived market/competition benefits and perceived operational benefits are positively related to traceability systems implementation and readiness for HAS. It was found that traceability systems are fully needed to bridge the connection between perceived benefits and readiness for HAS. This paper has important implications for resource-based theory, literature on HAS and traceability, SMEs, and halal authorities and certifying bodies. Limitations and directions for future studies are provided.
\end{abstract}

Keyword: Food manufacturers; Halal Assurance System; Traceability; Operational benefits; Market benefits 